\title{
Infra-Auricular Flap for Single Stage Ear Lobe Reconstruction
}

\author{
KHALED M. HASSAN, M.D. \\ The Department of Plastic and Reconstructive Surgery, Faculty of Medicine, Minia University, Egypt
}

\begin{abstract}
Background: Ear lobe deformity may be congenital or more often acquired due to trauma, burns or surgery. Losing them represents an obvious aesthetic abnormality. Its surgical repair places a challenge due to the difficulty of obtaining a natural appearing and durable outcome. Several methods developed to reconstruct this deformity have presented various advantages and disadvantages. We present the results of a relatively simple technique to reconstruct ear lobe in a single stage.
\end{abstract}

Patients and Methods: In this study, we used proximally based infra auricular flap to reconstruct 5 ear lobes in the period from February 2015 to July 2016 in Plastic Surgery Department, Minia University Hospital and private sector. Surgical procedure was performed under local anaesthesia in all cases. Except one case, we followed cases for 6 months post-operatively.

Results: Mean operative time was 40 minutes. Mean age was 32 years. All flaps survived. One patient required secondary sutures for partial ear wound dehiscence due to early removal of stitches.

Conclusion: The author outlines a simple method for reconstructing the entire lobe in one stage, without grafts, whilst offering a pleasant appearance with minimal donor site morbidity. The technique is suggested to reconstruct all cases of ear lobe defects.

Key Words: Infra-auricular - Ear lobe - Reconstruction.

\section{INTRODUCTION}

The earlobe is an anatomical structure of small dimensions without specific function, but with a significant aesthetic role. With an abundant blood supply and without cartilaginous tissue, the challenge underlying its reconstruction is related to the difficulty in obtaining a longstanding and aesthetically acceptable outcome. Almost every reconstructive method for total earlobe repair implies the use of adjacent tissue to compose a pedicled or bilobed flap. The residual deformity, location of the scars and the viability of the neighboring tissue are the key factors that determine the selection of the reconstructive method. There are several techniques described for that purpose, and all can be performed with local anesthesia. Gavello et al., in 1907, was one of the first authors to describe a method using a bilobed flap with an anterior base, located beneath the auricular defect [1]. The flap is raised and folded upon itself, horizontally, with the anterior flap forming the anterior part of the reconstructed earlobe. Brent's technique is one example of that, used for larger defects of the lower third of the auricle and earlobe, resorting to fold-over flaps and cartilaginous grafts to give volume and shape to the new earlobe [2]. Brent recommended the increment of the flap by over $30 \%$ and resorting to cartilaginous grafts (contralateral auricle or chondrocostal cartilage) to oppose tissue contraction.

More recently, in 1991, Seidman and Novelly described a single stage technique, with a U-shaped flap below the auricle, drawn inferiorly to the lower part of the earlobe [3]. The flap is then raised, keeping its pedicle, tubularized over itself vertically (to shape and provide volume) creating a medial surface to the reconstructed earlobe. The postauricular closure is in $\mathrm{V}-\mathrm{Y}$, closing the donor area beneath the auricle. The size of the reconstructed earlobe may fluctuate, according to this technique, changing the length and width of the U flap. The present technique is different from the above mentioned techniques. We used an infra-auricular flap that is folded on itself horizontally with direct straight closure of donor area in single stage.

\section{PATIENTS AND METHODS}

Five patients were operated upon in the period from February 2015 to July 2016 in Plastic Surgery Department, Minia University Hospital and private sector.

\section{Surgical technique:}

All surgeries were done under local anaesthesia using lidocaine (2\%) with adrenalin 1:100,000. Superiorly based random pattern infra-auricular flap was raised after tracing the defect template from the normal side. Half of the flap was taken from pre-auricular skin and the other from postauricular skin (Fig. 1). 

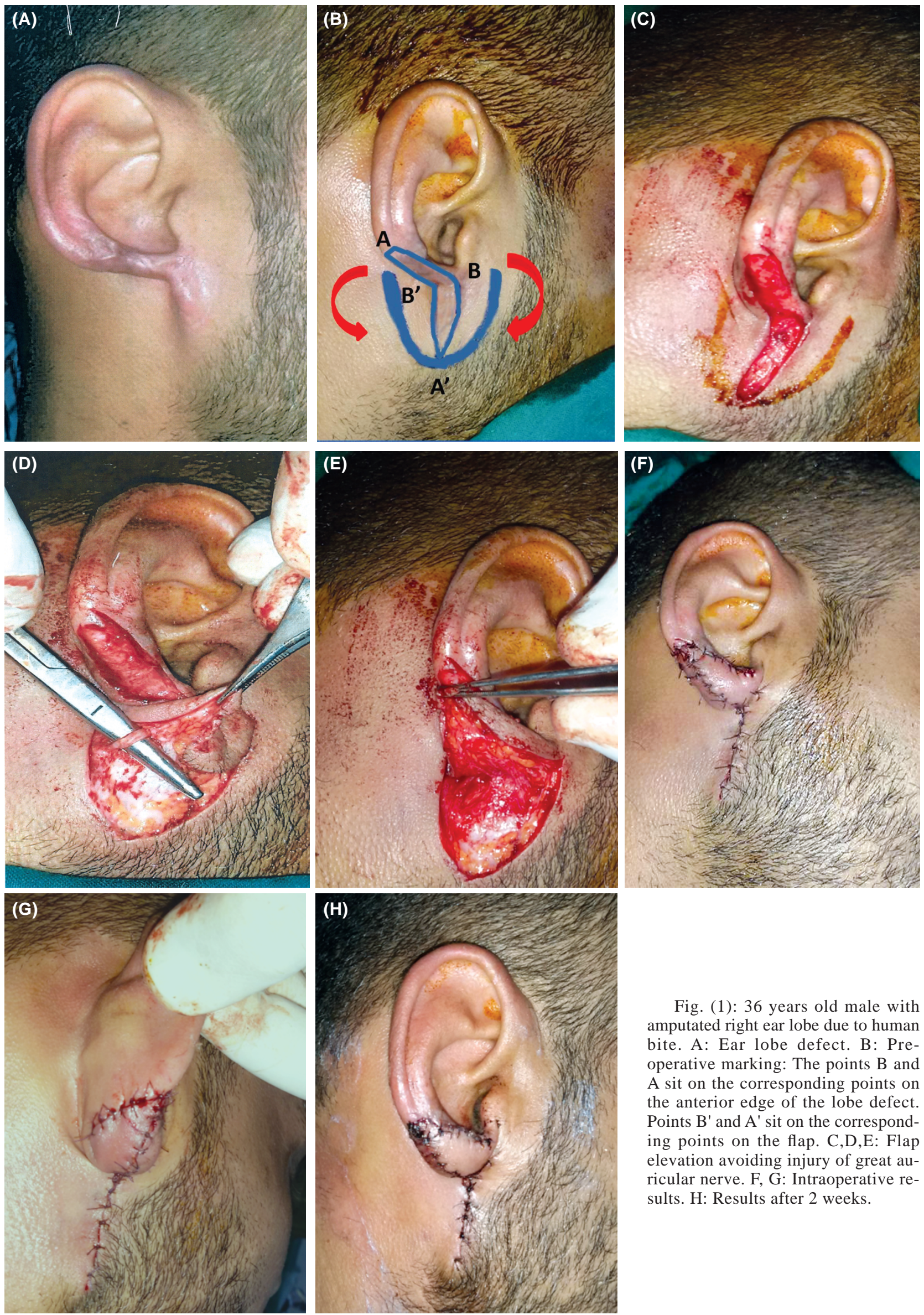

Fig. (1): 36 years old male with amputated right ear lobe due to human bite. A: Ear lobe defect. B: Preoperative marking: The points B and A sit on the corresponding points on the anterior edge of the lobe defect. Points B' and A' sit on the corresponding points on the flap. C,D,E: Flap elevation avoiding injury of great auricular nerve. F, G: Intraoperative results. H: Results after 2 weeks. 
Anterior and posterior margins of the lobule defect are marked and ends are labelled as A and B. Corresponding flap area ends is marked as A' $B^{\prime}$. Creation of raw surface along these lines by de-epithelization was done. Flap was elevated in the subcutaneous plane preserving the subdermal vascular plexus. Careful dissection should be done to avoid injury of great auricular nerve which lies underneath the flap. Flap wings sutured to each other to form the infero-lateral border of the new ear lobe. Raw areas created on flap and auricle sutured together. Donor site was closed in two layers primarily after undermining. Patients were instructed to avoid smoking and cold exposure.

\section{RESULTS}

Age of patients ranged from 23 to 46 years old (mean 32 years old). Four patients were males and one female. All patients had complete loss of ear lobe. Four were due to human bites and one due to sharp injury to the lobe with a knife. All presented after an average of 3-4 months post injury except one who presented acutely.

All but one patients followed-up for 6 months post-operative. All patients had uneventful postoperative period apart from one patient who required secondary sutures to disrupted flap edge. Flap had enough soft tissue to provide bulk without the use of cartilage grafts. No flap shrinkage was noted in any of the cases and the contour of the lobe was maintained over six months as well Fig. (2).
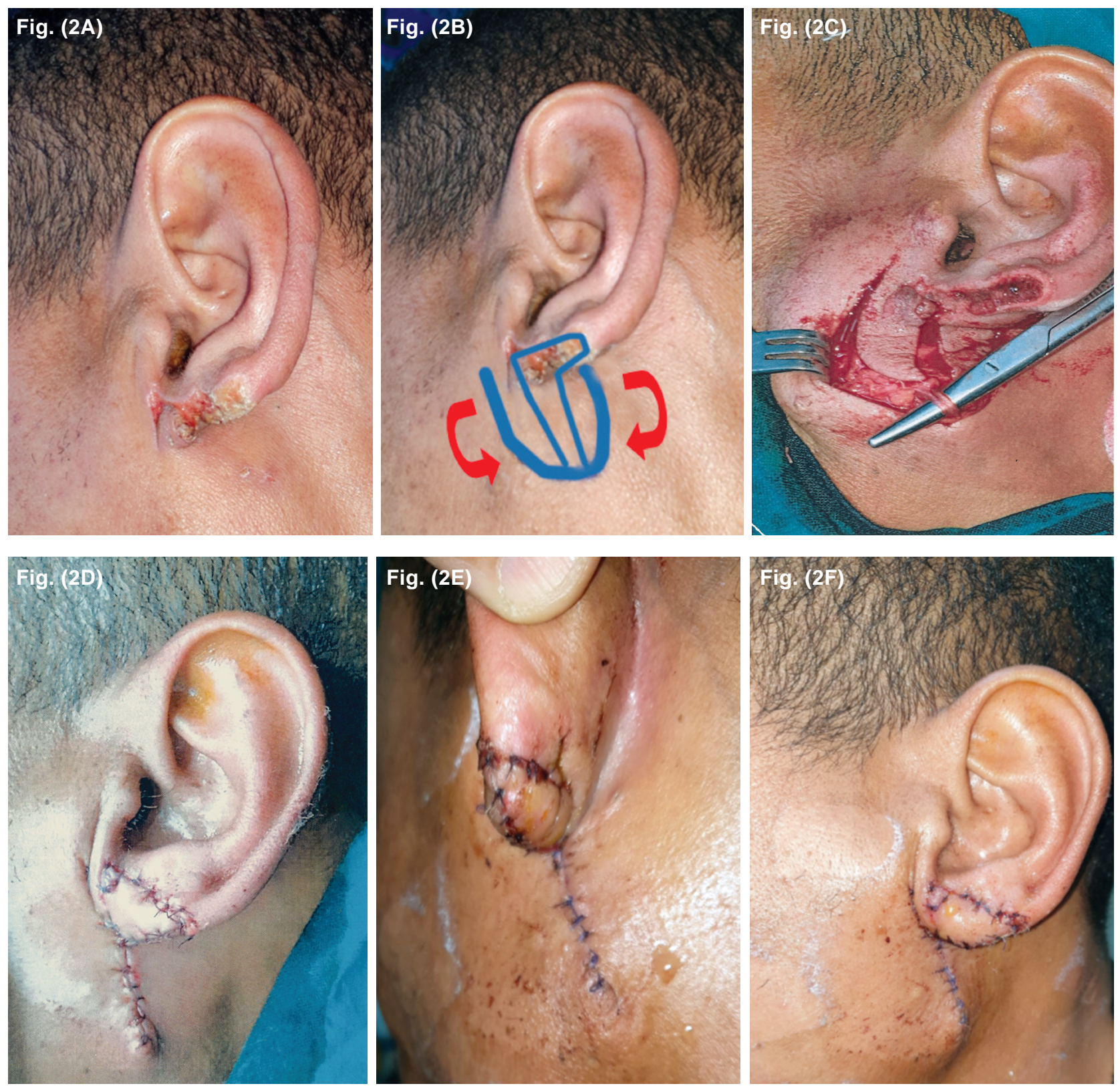

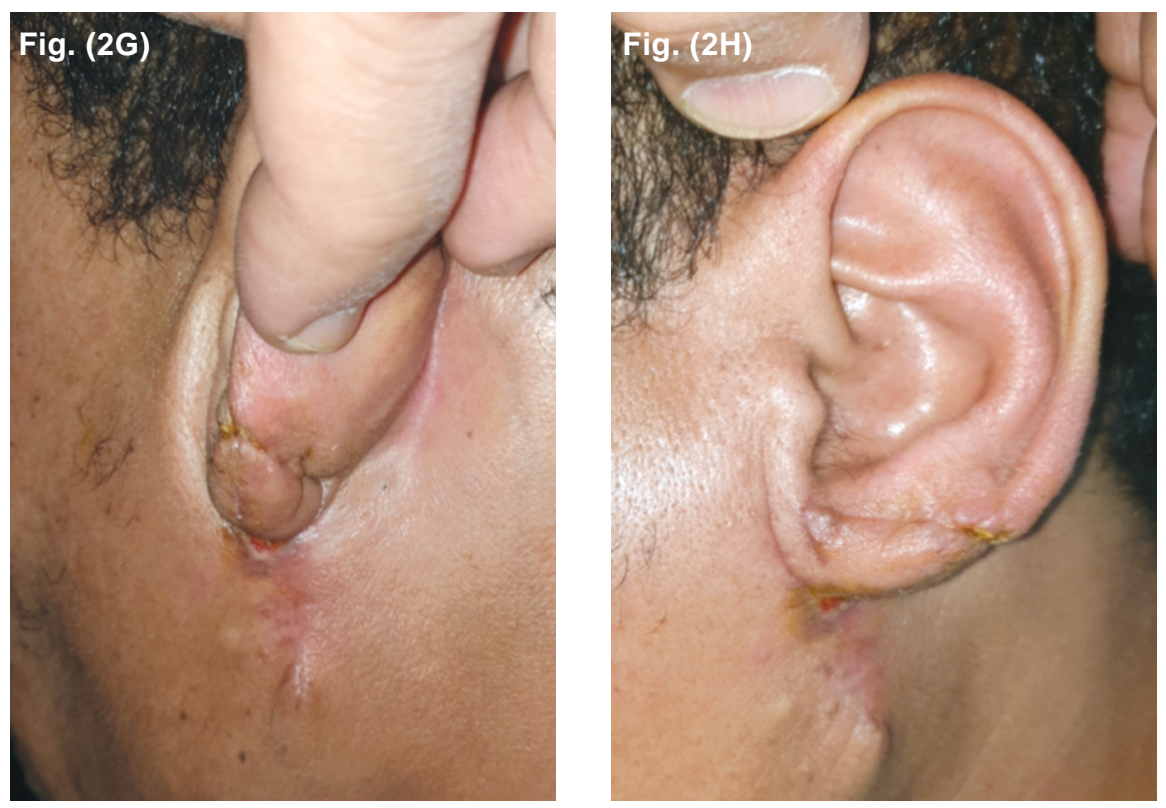

Fig. (2): 42 years old male with amputated left ear lobe due to human bite. A: Ear lobe defect. B: Pre-operative marking. C: Flap elevation avoiding injury of great auricular nerve. D: Intraoperative results. E,F: Results after 2 weeks. G,H: Results after 2 months.

\section{DISCUSSION}

The aesthetic importance of the normal ear lobe was recognised as early as $900 \mathrm{BC}$, as accounted by Converse who quoted, the Susruta, an Indian text of ancient medicine, $900 \mathrm{BC}$, where partial reconstruction of the earlobe was done with a cheek flap [4].

Different techniques were reported to reconstruct ear lobe. Preaux in 1972 reported the use of superiorly based flap double don itself to repair lost earlobe [5]. Brent in 1976 reported the use of modified "valise handle" technique using contralateral conchal cartilage grafts that are implanted subcutaneously and later raised as bipedicled chondrocutaneous flaps to adequately reconstruct the lower third of the ear [2]. Davies in 1974 described a technique that uses a posterior (medial) lining flap for anterior (lateral) earlobe reconstruction [6]. Our procedure involves a single stage reconstruction of earlobe with the aid of superiorly based flap folded on itself and inset into the defect (Fig. $1)$. The secondary defect is closed primarily with the scar being hidden in the cervicofacial crease behind the ramus of the mandible. The bulk of the soft tissue in the flap prevents shrinkage of the flap, thus avoiding the need for a cartilage graft. It provides a rounded contour to the ear lobe, a desired feature which is often difficult to produce with other methods.

\section{Concluson:}

The above mentioned technique of ear lobe reconstruction offers a relatively simple method that is performed in a single stage. It provides a pleasant rounded contour and good bulk without the need for cartilage graft augmentation. The results are durable and donor site morbidity is minimal as well. The study suggests that the above mentioned technique is suitable to reconstruct all cases of ear lobe defects.

\section{REFERENCES}

1- Gavello P. and Les autoplasties: Paris: G. Steinheil., [Cite' par Nelaton C, Ombredanne L], 1907.

2- Brent B.: Earlobe construction with an auriculo-mastoid flap. Plast. Reconstr. Surg., 57: 389-91, 1976.

3- Seidman M.D. and Novelly N.J.: Creation of a lobule: A simple technique. Otolamgol., FINS 105: 734-7, 1991.

4- Converse J.M.: Reconstruction of the auricle: 1. Plast. Reconstr. Surg. Transpl. Bull., 22: 150-63, 1958.

5- Preaux J.: A simple procedure for reconstruction of the lower part of the auricle [in French]. Ann. Chir. Plast., 16: 60-2, 1971.

6- Davis J.: Symposium on reconstruction of the auricle. St. Louis: CV Mosby Company, 1-312, 1974. 